\title{
Condiciones locales de vulnerabilidad asociadas con dengue en dos comunidades de Morelos
}

\author{
Silvia Chuc, MSP,(I) Magali Hurtado-Díaz, MC,(I) Astrid Schilmann, MC, (I) Horacio Riojas-Rodríguez, PhD, (I) \\ Hilda Rangel, MC, ${ }^{(2)}$ Mariana Irina González-Fernández, MC.(3)
}

Chuc S, Hurtado-Díaz M, Schilmann A, Riojas-Rodríguez H, Rangel H, González-Fernández MI. Condiciones locales de vulnerabilidad asociadas con dengue en dos comunidades de Morelos. Salud Publica Mex 2013; 54: 170-178.

\begin{abstract}
Resumen
Objetivo. Evaluar las condiciones de vulnerabilidad asociadas con la ocurrencia de dengue en dos localidades de Morelos en los años 2006 a 2009. Material y métodos. se aplicó una encuesta sobre conocimientos, percepción de riesgo, prácticas de prevención y uso del agua en dos localidades de Morelos. Mediante un análisis de componentes principales, se construyó un índice de vulnerabilidad local al dengue (IVL). La asociación del IVL con la enfermedad en la vivienda se evaluó mediante una prueba de ji cuadrada. Resultados. El IVL incluyó cinco componentes explicando el $63 \%$ de la varianza y fue clasificado en tres categorías: baja, media y alta. Se observó una asociación significativa entre el aumento de la vulnerabilidad y la prevalencia del reporte de casos de dengue en las localidades. Conclusión. El estudio de la vulnerabilidad al dengue permite identificar necesidades locales específicas en materia de promoción de la salud.
\end{abstract}

Palabras clave: vulnerabilidad en salud; dengue; Morelos; México
Chuc S, Hurtado-Díaz M, Schilmann A, Riojas-Rodríguez H, Rangel H, González-Fernández MI.

Local conditions of vulnerability associated

with dengue in two communities of Morelos.

Salud Publica Mex 2013; 54: I70-178.

\section{Abstract}

Objective. To evaluate the vulnerability associated with the occurrence of dengue in two villages of Morelos, Mexico from 2006 to 2009. Materials and methods. A survey on knowledge, risk perception, prevention practices and water use was applied in two villages of Morelos. Using a principal component analysis, an index of local vulnerability to dengue (IVL) was constructed. The association of IVL with the disease at home was assessed using a Chi-square test. Results. The IVL included five components explaining $63 \%$ of the variance and was classified in three categories: low, medium and high. There was a significant association between increased vulnerability and prevalence of reported cases of dengue in Temixco and Tlaquiltenango. Conclusion. The study of vulnerability to dengue allows us to identify local needs in the field of health promotion.

Key words: health vulnerability; dengue; Morelos; Mexico

(I) Centro de Investigación en Salud Poblacional, Instituto Nacional de Salud Pública. Cuernavaca, Morelos, México.

(2) Centro de Investigación sobre Enfermedades Infecciosas, Instituto Nacional de Salud Pública. Cuernavaca, Morelos, México.

(3) Centro Regional de Control de Vectores de Cuautla, Servicios de Salud de Morelos. Cuautla, Morelos, México.

Fecha de recibido: 28 de mayo de 2012 - Fecha de aceptado: 23 de octubre de 2012 Autor de correspondencia: M.C. Magali Hurtado-Díaz. Instituto Nacional de Salud Pública. Av. Universidad 655, col. Santa María Ahuacatitlán. 62100 Cuernavaca, Morelos, México. Correo electrónico: mhurtado@insp.mx 
E dengue es una enfermedad transmitida por vector (ETV); la ocasiona un virus de la familia Flaviviridae y es transmitida por el mosquito Aedes aegypti. Hoy en día se reconocen cuatro serotipos: DEN-1, DEN-2, DENV-3, DENV-4. Cada año, alrededor de 50 a 100 millones de personas en el mundo son infectadas con este virus; de éstas, entre 250000 y 500000 se convierten en casos de fiebre hemorrágica por dengue (FHD) y 25000 fallecen. $^{1}$

En América Latina, las precarias condiciones de saneamiento básico y la urbanización no planificada han agravado el problema. En México, la fiebre por dengue (FD) comenzó a presentarse en 1978; en 1980 y en 1997 se registraron las cifras más elevadas de personas infectadas: 51406 y 52561 , respectivamente. ${ }^{2}$

El 1983, el estado de Morelos comenzó a notificar casos de FD y registró su primer brote en 1998. En 2006 ocurrió un segundo brote, con 2784 casos notificados, de los cuales $197 \%$ ocurrió en el sur y el oriente del estado afectando, entre otros, al municipio de Tlaquiltenango. ${ }^{3}$ En 2008 se notificaron 8118 casos que representan el brote de mayor magnitud registrado en la entidad; $82 \%$ de los casos se localizó en el norte afectando, entre otros, al municipio de Temixco. ${ }^{4}$

\section{Enfoque de vulnerabilidad}

La vulnerabilidad es un concepto multidimensional y su operacionalización implica una tarea interdisciplinaria. A diferencia de la evaluación de riesgos, involucra, al menos: a) la exposición, esto es, el contacto de la población con un peligro en particular; b) la sensibilidad, es decir, el grado en el que está afectada la población por la exposición a otros peligros, y c) la resiliencia o habilidad de la población para resistir o recuperarse del daño asociado con los múltiples peligros. ${ }^{5}$

En términos generales, una mayor vulnerabilidad se vincula con poblaciones que experimentan mayor exposición y cuentan con poca capacidad para enfrentar o recuperarse del peligro. Dicha capacidad varía de una población a otra y, aunque las comunidades enfrenten una misma amenaza, podrían no ser igualmente vulnerables. ${ }^{6}$

En el análisis interdisciplinario de las ETV, la vulnerabilidad define la exposición como la presencia del vector o antecedente de la enfermedad; la sensibilidad incluye las condiciones sociales de la población, y la resiliencia es la capacidad de respuesta en función de los recursos disponibles para evitar enfrentar la enfermedad, siendo relevantes las actitudes y prácticas de la población, los factores ideológicos, culturales y educativos vinculados con la percepción de riesgo y la generación de herramientas prácticas y conceptuales para hacer frente a la enfermedad. ${ }^{7}$

El presente estudio propone una selección de variables mínimas para evaluar la vulnerabilidad comunitaria a la ocurrencia de dengue en función de los conocimientos, actitudes, costumbres y percepción de riesgo, con información correspondiente a los años comprendidos entre 2006 y 2009, en dos localidades del estado de Morelos.

\section{Material y métodos}

\section{Diseño y población de estudio}

Este estudio transversal se originó a partir del diagnóstico comunitario y el establecimiento de prioridades con respecto a los problemas de salud en la colonia Gabriel Tepepa, del municipio de Tlaquiltenango. Se seleccionó, además, la colonia Lomas del Carril, ubicada en el municipio de Temixco, como una localidad de comparación, de acuerdo con los datos epidemiológicos de los servicios de salud del estado de Morelos (SSM).

La colonia Gabriel Tepepa, la más grande de la localidad de Tlaquiltenango $\left(18^{\circ} 37^{\prime} 57^{\prime \prime} \mathrm{N}, 99^{\circ} 9^{\prime} 46^{\prime \prime} \mathrm{O}\right)$, presenta un grado de marginación bajo. Tiene 6567 habitantes y se encuentra rodeada por campos de cultivo de caña; la atraviesan varios canales de riego y descargas domiciliarias. La colonia Lomas del Carril, en Temixco $\left(18^{\circ} 52^{\prime} 2.76^{\prime \prime} \mathrm{N}, 99^{\circ} 14^{\prime} 8.15^{\prime \prime} \mathrm{O}\right)$, tiene un grado de marginación alto. Con 8017 habitantes, se ubica entre dos barrancas cuyo cauce arrastra agua contaminada, descargas domiciliarias y residuos sólidos. ${ }^{8}$

En los meses comprendidos entre octubre de 2009 y marzo de 2010, se aplicó una encuesta a viviendas seleccionadas mediante muestreo aleatorio sistemático con reemplazo, recolectando información retrospectiva del periodo 2006 a 2009. Un total de 348 viviendas en Gabriel Tepepa y de 165 en Lomas del Carril conformaron la muestra, cuyo tamaño se definió de acuerdo con la capacidad logística del estudio. El Comité de Ética del Instituto Nacional de Salud Pública aprobó el protocolo del estudio.

\section{Recolección de la información}

Se aplicó la encuesta a cada jefe de familia, previa obtención de su consentimiento verbal. La encuesta incluyó las siguientes categorías: agua y residuos (tres variables); conocimiento de la enfermedad y el vector (siete variables); percepción de riesgo (dos variables) y medidas de prevención (seis variables). En la figura 1 se muestran las variables, así como el elemento de vulnerabilidad al que corresponde cada una. 

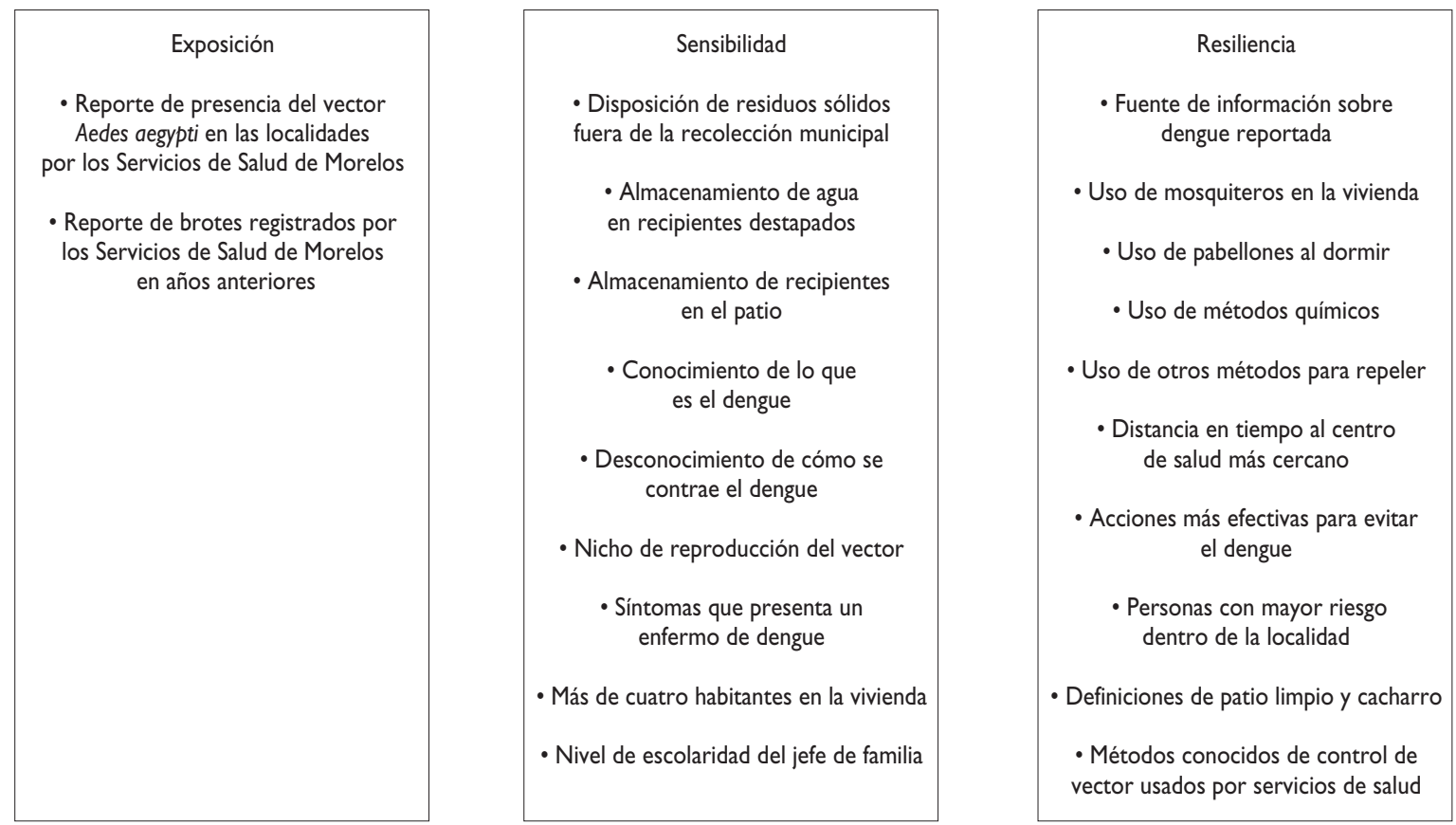

Figura I. Dimensiones de la VULnerabilidad local al dengue INCLUidas EN LA ENCUESTA APLiCADA EN LOS MUNICIPIOS de Tlaquiltenango y Temixco en los años 2009 y 2010. Morelos, México

\section{Análisis estadístico}

Se obtuvieron 22 variables categóricas, cuyas opciones de respuesta fueron codificadas con valores de 0 a 3, de modo que un valor mayor expresara una condición más desfavorable. Las variables de respuesta fueron una dicotómica, que indicaba la presencia de, al menos, un caso de dengue en la vivienda (presencia de la enfermedad) y una de conteo, con el número de casos presentados en la vivienda, ambas para un periodo que iniciaba en el año 2006 y llegaba hasta el momento de la aplicación de la encuesta.

Se realizaron un análisis descriptivo para todas las variables, una comparación de proporciones entre ambas localidades por medio de una tabla comparativa, y una prueba ji cuadrada. Posteriormente se realizó un análisis bivariado entre las variables dependientes, con cada una de las variables respuesta; con la correspondiente a presencia de la enfermedad, se aplicó una regresión logística simple y se obtuvieron razones de momios de prevalencia $(R M p)$, mientras que para el número de casos se llevó a cabo una regresión simple de tipo binomial negativa dada la sobre dispersión de los datos y debido al hecho de que se observó un máximo de 10 casos en la vivienda; en este caso, se obtuvieron razones de prevalencias $(R P){ }^{9}$

De esta manera, se seleccionaron las variables para construir el índice de vulnerabilidad local (IVL) al dengue, considerando aquellas que resultaron significativas en los modelos de regresión simple (valor de corte $p<0.10$ ).

\section{Índice de vulnerabilidad local al dengue}

Mediante el análisis de componentes principales (ACP), se redujo el conjunto de 11 variables seleccionadas a un menor número de dimensiones. Se obtuvo la matriz de correlación para datos categóricos, y la selección del número de componentes estuvo en función de que el valor propio (eigenvalor) fuera mayor a 1 , y el porcentaje de variabilidad explicado por cada uno de los componentes, y en conjunto, fuese el mayor posible. ${ }^{10,11}$

Dentro de cada componente, las variables dominantes, es decir las de mayor valor, fueron identificadas a través de los vectores de carga (eigenvector) y se estableció un punto de corte de \pm 0.40 .

Una vez seleccionados los componentes, se generó una puntuación para cada uno de ellos; los valores 
continuos se categorizaron en tres niveles, tomando como puntos de corte los percentiles 25 y 75 , de acuerdo con la metodología propuesta por Camponovo. ${ }^{12}$ Los puntajes de cada componente tomaron valores 1, 2 o 3 , donde una mayor puntuación indicaba un mayor nivel de vulnerabilidad. Finalmente, las puntuaciones de todos los componentes se sumaron, de tal manera que se obtuvo la cifra compuesta del IVL al dengue y cada vivienda quedó clasificada dentro de una categoría de vulnerabilidad baja, media o alta.

Este modelo aditivo no consideró ningún supuesto a priori sobre la ponderación de cada componente en la suma de los mismos, puesto que no existen antecedentes para determinar la importancia de cada grupo de indicadores. ${ }^{13}$

Por último, para evaluar la relación entre el IVL y la presencia de la enfermedad, se realizó una comparación de proporciones a través del estadístico de prueba ji cuadrada.

\section{Resultados}

\section{Características de las viviendas encuestadas}

De las 530 viviendas seleccionadas, 17 (3.3\%) no fueron encuestadas debido a la ausencia de un adulto en el hogar. En total se encuestaron 348 viviendas en Gabriel Tepepa y 165 en Lomas del Carril; la proporción de no respuesta fue de $10(2.9 \%)$ y siete $(4.2 \%)$, respectivamente. Las personas entrevistadas fueron, en su mayoría, del sexo femenino; la media de habitantes por vivienda en ambas colonias fue de cuatro personas.

En Lomas del Carril 40\% de las viviendas tuvo, al menos, un caso de dengue en el periodo de estudio, mientras que en Gabriel Tepepa la cifra ascendió a 45\%. Por otra parte, $70 \%$ declaró contar con algún tipo de servicio de salud (cuadro I)

\section{Vulnerabilidad local al dengue}

En la comparación de proporciones, se encontró una diferencia significativa entre las dos localidades para seis variables de la categoría de conocimiento, dos en la categoría de agua y residuos, dos de percepción de riesgo y una en la de medidas de prevención y protección (cuadro II).

En el análisis de regresión, las 11 variables que se asociaron significativamente $(p<0.10)$ con la presencia de la enfermedad en alguna de las dos colonias o en toda la muestra fueron: presencia de más de cuatro habitantes en la vivienda (RMp = 1.40; IC95\% 0.99-1.99); distancia en tiempo entre la vivienda y el centro de salud donde se atienden (RMp=1.53; IC95\% 1.17-1.99);

\section{Cuadro I}

DESCRIPCIÓN DE LA POBLACIÓN DE ESTUDIO SOBRE CONDICIONES DE VULNERABILIDAD ASOCIADAS CON DENGUE, ( $\mathrm{N}=5$ I 3 VIVIENDAS) EN LOS MUNICIPIOS DE Tlaquiltenango y Temixco. estado de Morelos, MÉXICO, 2006-2009

\begin{tabular}{|c|c|c|}
\hline Variables & $\begin{array}{c}\text { Gabriel Tepepa } \\
n(\%)\end{array}$ & $\begin{array}{c}\text { Lomas del Carril } \\
n(\%)\end{array}$ \\
\hline Viviendas encuestadas & 348 & 165 \\
\hline \multicolumn{3}{|l|}{ Total de habitantes en las viviendas } \\
\hline encuestadas & 1650 & 770 \\
\hline Más de cuatro habitantes en la vivienda & $160(46)$ & $88(53)$ \\
\hline Informantes de sexo femenino & $247(7 I)^{*}$ & $137(83)^{*}$ \\
\hline \multicolumn{3}{|l|}{ Nivel de escolaridad del informante } \\
\hline Sin estudios & $73(2 I)^{*}$ & $77(46)^{*}$ \\
\hline Básico & 275 (79) & $88(54)$ \\
\hline
\end{tabular}

Habitantes de la vivienda cuentan

con algún servicio de salud $\quad 249(72) \quad 120(73)$

Viviendas con al menos un caso

de dengue en el periodo $\quad$ 151 (43) 67 (4I)

\begin{tabular}{lll}
\hline Total de casos notificados de FD & 207 & 106
\end{tabular}

\begin{tabular}{lll}
\hline Total de casos notificados de FHD & 22 & 19 \\
\hline
\end{tabular}

Prevalencia de FD (casos por 100 habitantes) $^{12}$

Prevalencia FHD§ (casos por 100 habitantes) । 2

* Diferencia de proporciones entre localidades $(p<0.05)$

¥FD: fiebre por dengue

$\S$ FHD: fiebre hemorrágica por dengue

almacenamiento de agua en recipientes destapados $(\mathrm{RMp}=2.94$; IC95\% 1.46 - 5.91); almacenamiento de recipientes en el patio (RMp=1.55; IC95\%1.03-2.34); desconocimiento de la forma como se contrae el dengue $(\mathrm{RMp}=1.56$; IC95\% 1.06-2.29), y falta de mosquiteros en la vivienda (RMp=1.77; IC95\% 0.92-3.43). Con el número de casos en la vivienda, se asociaron significativamente las siguientes variables: nivel de escolaridad básico ( $\mathrm{RP}=0.73$; IC95\% 0.53-0.94); el uso de pabellones $(\mathrm{RP}=0.80$; IC95\%: 0.65 - 0.98); disposición de residuos fuera de la recolección municipal $(\mathrm{RP}=1.08$; IC95\% 0.55-2.12); fuente de donde obtuvo la información ( $\mathrm{RP}=1.10$; IC95\% 0.98-1.24) y personas con más riesgo en la localidad ( $\mathrm{RP}=1.12$; IC95\% 0.98 - 1.27).

\section{Construcción del índice de vulnerabilidad local al dengue}

Se seleccionaron cinco componentes acumulando 63\% de la varianza original de los datos (cuadro III). Median- 


\section{Cuadro II}

DesCRIPCIÓN DE LAS VARIABLES EMPLEADAS PARA EVALUAR VULNERABILIDAD AL DENGUe A PARTIR DE LA ENCUESTA APLICADA en los municipios de Tlaquiltenango y Temixco estado de Morelos, México, en 2009 y 2010

$\begin{array}{ccc} & \text { Gabriel Tepepa } & \text { Lomas del Carril } \\ \text { Variable } & n(\%) & n(\%)\end{array}$

Agua y residuos

Disposición de residuos sólidos fuera

de la recolección municipal

$10(3)^{*}$

II (7)*

Almacenamiento de agua en recipientes

destapados

$195(56)$

$105(64)$

Almacenamiento de recipientes en el patio $59(17)^{*}$

$63(38)^{*}$

Conocimiento

Qué es el dengue

Una enfermedad

$275(79) \quad 138(83)$

Un virus o bacteria

$38(1$

$9(5)$

Otros

$35(10)$

$18($ II)

Desconocimiento de cómo se contrae

el dengue

$135(39)^{*}$

$12(7)^{*}$

Nicho de reproducción del vector

\begin{tabular}{lcc} 
En el agua limpia & $152(44)^{*}$ & $124(75)^{*}$ \\
\hline En cacharros con agua & $66(19)$ & $7(4)$ \\
\hline Otros & $130(37)$ & $34(21)$
\end{tabular}

Síntomas que presenta un enfermo de dengue

Dolor de cabeza y de huesos, fiebre,

\begin{tabular}{lcc} 
escalofrío & $94(27)^{*}$ & $57(35)^{*}$ \\
\hline Uno de los anteriores & $181(52)$ & $61(37)$ \\
\hline No sabe & $73(21)$ & $4729)$
\end{tabular}

Fuente de información sobre dengue notificada por el informante

\begin{tabular}{lcc} 
Brigadistas & $216(62)^{*}$ & $67(4 I)^{*}$ \\
\hline Televisión & $40(12)$ & $67(4 I)$ \\
\hline Radio & $31(9)$ & $0(0)$ \\
\hline Ninguna & $61(17)$ & $31(18)$
\end{tabular}

Definición de patio limpio

Sin agua estancada o cacharros

\begin{tabular}{lll} 
Sin vegetación & $117(34)$ & $33(26)^{*}$ \\
\hline Barrido & $162(46)$ & $90(55)$
\end{tabular}

Variable

Gabriel Tepepa Lomas del Carril
$n(\%)$
$n(\%)$

Definición de cacharro

Recipientes que almacenan agua $\quad 42(12)^{*} \quad 42(25)^{*}$

\begin{tabular}{lcc}
\hline Botes de plástico & $86(25)$ & $47(29)$ \\
\hline No sabe & $220(63)$ & $76(46)$
\end{tabular}

Métodos conocidos de control de vector usados por los servicios de salud

Todos (nebulización, control larvario,

control biológico, descacharrización) $249(71)^{*} \quad 0(0)^{*}$

\begin{tabular}{lcc}
\hline Tres de las anteriores & $67(19)$ & $133(8 \mathrm{I})$ \\
\hline Dos de las anteriores & $8(2)$ & $19(\mathrm{II})$ \\
\hline Ninguna & $24(7)$ & $13(8)$
\end{tabular}

Percepción de riesgo

Acciones más efectivas en el control del vector

Todas (nebulización, control larvario,

\begin{tabular}{lcc} 
control biológico, descacharrización) & $0(0)^{*}$ & $47(28)^{*}$ \\
\hline Tres de las anteriores & $72(2 \mathrm{I})$ & $0(0)$ \\
\hline Dos de las anteriores & $228(65)$ & $107(65)$ \\
\hline Ninguna & $48(14)$ & $11(7)$
\end{tabular}

Personas con mayor riesgo de enfermar dentro de la localidad

\begin{tabular}{lcc} 
Todos & $221(63)^{*}$ & $77(47)^{*}$ \\
\hline Los que son sucios & $6 I(18)$ & $19(12)$ \\
\hline Niños y ancianos & $22(6)$ & $69(4 I)$ \\
\hline No sabe & $44(13)$ & $0(0)$
\end{tabular}

Medidas de prevención y protección

\begin{tabular}{lll} 
Uso de mosquiteros en la vivienda & I34 (39) & $54(33)$ \\
\hline Uso de pabellones al dormir & $221(63)$ & $116(70)$ \\
\hline Uso de métodos químicos & $222(64)$ & $102(62)$ \\
\hline $\begin{array}{l}\text { Uso de otros métodos para repeler } \\
\text { el mosquito }\end{array}$ & $91(52)$ & $26(4 I)$
\end{tabular}

Distancia en tiempo al centro de salud *

\begin{tabular}{lcc} 
Menos de 15 minutos & $155(45)$ & $86(52)$ \\
\hline Entre 15 a 30 minutos & $164(47)$ & $44(27)$ \\
\hline Más de 30 minutos & $29(8)$ & $35(21)$
\end{tabular}

* Diferencia de proporciones entre localidades $(p<0.05)$ 


\section{Cuadro III \\ SELECCIÓn de VARIABLES dominantes en CADA COMPONENTE PARA CONSTRUIR EL ÍNDICE DE VULNERABILIDAD LOCAL AL dengue de la encuesta aplicada en los municipios de Tlaquiltenango y Temixco estado de Morelos, México, EN 2009 Y 2010}

\begin{tabular}{ccccc} 
Componente & \% de varianza explicada & Variables dominantes & Vector de carga \\
\hline & 15.6 & Almacenamiento de agua en recipientes destapados & 0.519 & 0.464 \\
\hline 2 & 14.3 & Almacenamiento de recipientes en el patio & 0.694 \\
& & Disposición de residuos sólidos fuera de la recolección municipal & 0.409 \\
\hline 3 & 12.3 & Nivel de escolaridad del jefe de familia & -0.531 \\
& & Uso de pabellones al dormir & 0.505 \\
\hline 4 & 11.2 & Más de cuatro habitantes en la vivienda & 0.489 \\
\hline 5 & & Distancia en tiempo al centro de salud & 0.637 \\
& 9.8 & Desconocimiento acerca de cómo se contrae el dengue & 0.561 \\
\hline
\end{tabular}

te la prueba de Kaiser-Meyer-Olkin (0.52), se observó que la muestra no es adecuada, pues las variables no presentaron una correlación tan alta como se hubiera esperado. En el índice quedaron representadas seis variables pertenecientes al elemento sensibilidad y cinco correspondientes a resiliencia.

Del total de la muestra, $215(41 \%)$ viviendas fueron ubicadas en el nivel bajo, 189 (37\%) en el medio y 109 (22\%) en el alto. Lomas del Carril presentó un mayor porcentaje (28\%) del total de viviendas encuestadas en el nivel alto de vulnerabilidad, mientras que en Gabriel Tepepa sólo 17\% de las viviendas encuestadas se ubicaron en dicho nivel; esa diferencia fue significativa $(p<0.05)$.

Como se observa en la figura 2, existe una mayor proporción de viviendas con presencia de la enfermedad, en la medida en que aumentan los niveles de vulnerabilidad. Esta relación resultó significativa para Lomas del Carril, aunque no así para Gabriel Tepepa. Además, la prueba resultó significativa $(p=0.01)$ cuando se utilizó el número de casos en toda la muestra y no para cada una de las colonias.

\section{Discusión}

En este estudio se seleccionaron variables locales de exposición, sensibilidad y resiliencia para evaluar la vulnerabilidad al dengue, utilizando ACP para resumir esta información en un IVL. Únicamente resultaron significativas algunas de las variables de cada categoría como el conocimiento del vector, la acumulación de recipientes en el patio de la vivienda y la acumulación insegura de agua, el nivel de escolaridad del jefe de familia y el hacinamiento en la vivienda. Las medidas para prevenir la picadura de los mosquitos -uso de insecticidas y mosquiteros- fueron similares en proporción en las dos localidades y sólo el uso de pabellones mostró una diferencia significativa.

Por otro lado, variables también consideradas importantes, como la cercanía a los servicios de salud y el conocimiento de las campañas de erradicación del vector, no mostraron significancia estadística y no se incluyeron en el IVL, aunque no se descarta su importancia.

Los índices para evaluar la vulnerabilidad asociada con dengue constituyen una herramienta útil para el abordaje cuantitativo. Algunos índices propuestos abarcan información sociodemográfica y ambiental, (Gómez, Schimdt, Lopes, Salazar, \& Achkart, 2008) permitiendo una evaluación regional. Otros índices se centran en variables contextuales como el conocimiento de la población sobre la enfermedad y el vector, el acceso a los servicios de salud y las medidas de prevención en la vivienda. ${ }^{14}$

En este estudio se encontró una relación significativa entre el alto nivel de vulnerabilidad al dengue medido a través del IVL y la presencia de la enfermedad en la vivienda. Quedó manifiesto que las variables de mayor peso son las relacionadas con el almacenamiento de agua, conocimiento de la enfermedad y el vector, medidas de prevención, percepción de riesgo y saneamiento básico, lo cual es congruente con otros estudios. ${ }^{15}$

En México, si bien las campañas de control del vector subrayan la importancia de acciones tales como lavar, tapar y voltear contenedores de agua para evitar criaderos, son escasos los programas integrales que contemplan los problemas de abastecimiento y pro- 
A) Presencia de la enfermedad en la vivienda

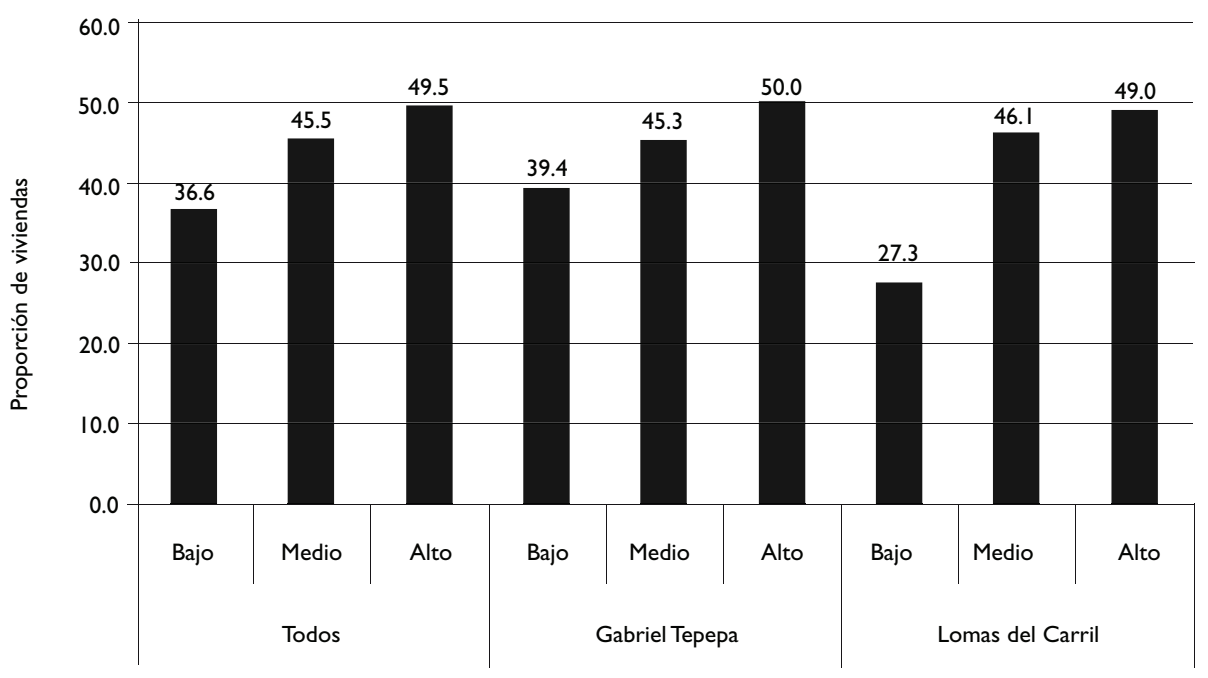

B) Dos o más casos reportados en la vivienda

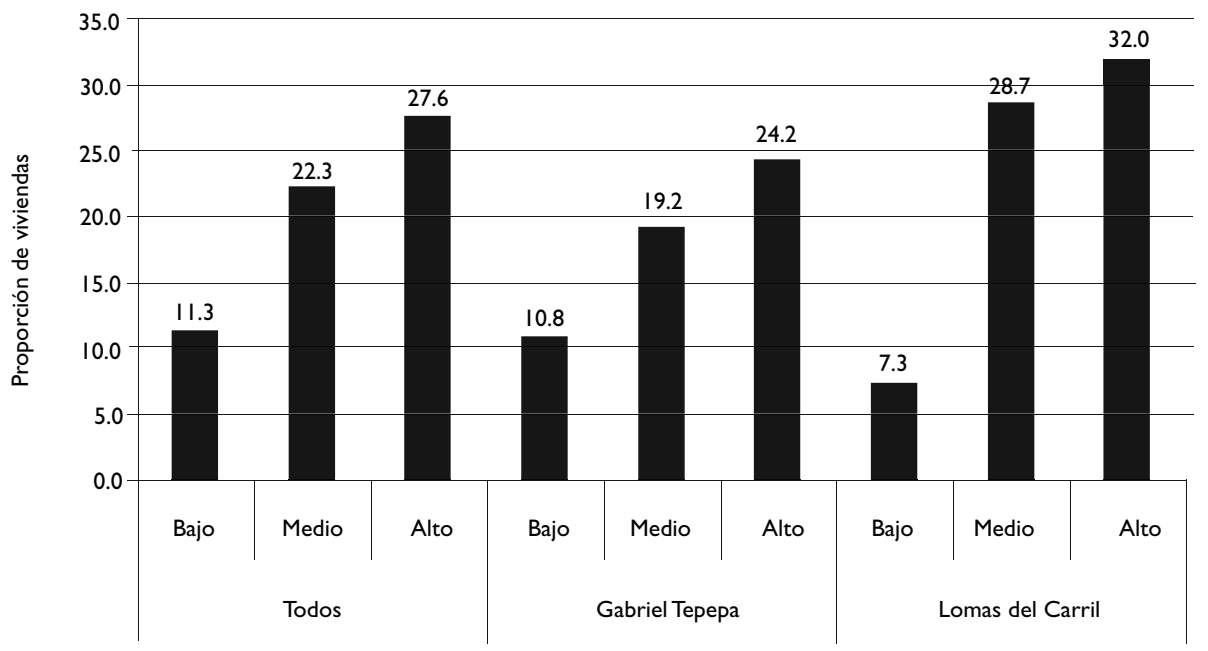

Figura 2. Casos de dengue notificados en la encuesta aplicada a la población de estudio en los municipios de TLAQuiltenango y Temixco, estado de Morelos, México en 2009 y 2010 , según el nivel de vulnerabilidad evaluado

mueven la práctica del almacenamiento seguro del agua. ${ }^{16}$ La capacidad de la población de enfrentar la enfermedad está parcialmente basada en el entendimiento y la percepción de la enfermedad y del vector. ${ }^{17}$ En este estudio se encontró una elevada prevalencia de desconocimiento acerca de la forma como se contrae el dengue, especialmente en Lomas del Carril, así como del nicho de reproducción del mismo -agua limpia-, lo cual podría asociarse con la ausencia o implementación incorrecta de medidas de prevención, influyendo así en los índices larvarios y de infestación domiciliaria del Aedes aegypti. ${ }^{18}$

En estudios que evalúan conocimientos sobre el vector se ha encontrado que los participantes pueden tener conocimientos adecuados acerca de la prevención del dengue, su sintomatología y tratamiento, pero también que no identifican al vector dentro de la vivienda como un factor riesgo de transmisión. Este desconocimiento 
del nicho, hábitos de alimentación y ecología del vector, limita el desarrollo y éxito de las medidas de prevención efectivas dentro de la vivienda. ${ }^{19}$

Las campañas de educación e información siguen siendo indispensables para la prevención de la enfermedad. Estudios llevados a cabo en México ${ }^{20}$ y Puerto Rico, ${ }^{21}$ han demostrado que las intervenciones educativas sobre características del vector, el conocimiento de la enfermedad y su relación con el desarrollo de medidas preventivas, están relacionadas con un cambio de actitud que resulta en una disminución de potenciales criaderos del vector. Asimismo, los resultados de un estudio desarrollado en Veracruz, México, corroboran -aunque en forma indirecta- este hecho, al notificar que los individuos con un bajo nivel de información presentaron casi dos veces más riesgo de enfermar en comparación con aquéllos que demostraron un conocimiento adecuado. ${ }^{22}$ En la colonia Gabriel Tepepa se observó un mayor desconocimiento acerca de la forma como se transmite la enfermedad, y en Lomas del Carril se detectó un menor conocimiento de los síntomas del padecimiento. Lo anterior indica que a pesar de que la población sea capaz de describir los síntomas de la enfermedad, en la práctica esos síntomas no se asocian con el dengue y llegan a confundirlos con padecimientos como la gripe, lo cual da lugar a una atención médica tardía y a una mayor probabilidad de que se presente un cuadro grave de FHD. ${ }^{23}$

Respecto a la percepción del riesgo de enfermar, los niños y los adultos mayores son percibidos como más susceptibles debido a su condición etaria. Asimismo, existe una asociación del concepto "suciedad" en la vivienda no asociada con la presencia del vector. La población percibe y reacciona ante los riesgos en salud según un proceso complejo modelado por experiencias previas, información, creencias y emociones, lo cual puede contribuir a un cambio de comportamiento a favor o en contra de la salud. ${ }^{24}$

Respecto a las medidas de prevención en el hogar, el uso de pabellones se asoció negativamente con la presencia de la enfermedad y los casos de dengue, lo cual es consistente con estudios desarrollados en Taiwán que muestran que el uso de pabellones y de mosquiteros en las ventanas disminuye hasta en $63 \%$ el riesgo de infección por dengue. ${ }^{25}$

El presente estudio muestra que existen varios factores que dificultan la participación social dentro del control del dengue. Si bien en ambas colonias la enfermedad fue identificada como un problema, existe una baja percepción de riesgo. Las acciones para su control provenientes del programa de vectores, en especial la nebulización, son percibidas como las más efectivas para la erradicación del dengue, mientras que el papel de la población dentro del control de la enfermedad no es claro.

Si bien este estudio transversal permitió generar hipótesis útiles para la planeación de los servicios de salud sobre algunas dimensiones de vulnerabilidad asociadas con la enfermedad en el contexto estudiado, por otro lado no permite estimar riesgos poblacionales y presenta limitaciones en cuanto al tamaño de la muestra, ya que debido a la escasez de tiempo y de recursos humanos no fue posible completar el tamaño de muestra estimado. Por otra parte, sólo se estudiaron dos localidades, lo cual impidió incluir el componente ambiental en la construcción del índice y no se consideraron todos los factores que pueden influir en la enfermedad. Sin embargo, no se conocen estudios que identifiquen factores de vulnerabilidad al dengue en México utilizando esta metodología. La selección de las colonias no fue aleatoria, considerando las recomendaciones de las autoridades sanitarias. Las variables analizadas no presentaron una correlación tan alta como se hubiera esperado, por lo que la construcción del índice estuvo limitada.

Si bien la metodología de ACP es útil en la construcción de índices, la agrupación de las variables dentro del análisis no parece responder a la lógica de los elementos que conforman la vulnerabilidad. No obstante, sí permite identificar aquellos elementos que probablemente desempeñan un papel en la sensibilidad y la resiliencia dentro de la población.

\section{Agradecimientos}

Esta investigación fue financiada por el Consejo Nacional de Ciencia y Tecnología (SALUD-2007-01-69933). Los autores agradecen a los Servicios de Salud de Morelos su significativa colaboración; a José Moreno Montoya y a Mariana Gómez Camponovo por sus valiosos comentarios, y a Jaqueline Martínez Avilés por el apoyo administrativo. Asimismo, desean expresar su reconocimiento a las autoridades y a los habitantes de cada comunidad donde se llevó a cabo la investigación por las facilidades otorgadas.

Declaración de conflicto de intereses. Los autores declararon no tener conflicto de intereses.

\section{Referencias}

I. World Health Organization. Dengue and Severe dengue. Fact Sheet $\mathrm{N}^{\circ}$ I 17. 20I2. who.int [sitio de internet] [actualizado 2012 enero; consultado 2012 marzo 13 ] Disponible en: http/www.who.int/mediacentre/ factsheets/fs I I7/en/index.html.

2. CINAVE. Casos por entidad federativa de enfermedades transmisibles por vector hasta la semana epidemiológica 5 I del 2009. México: Secretaría de Salud, 2009. 
3. SSM. Registro epidemiológico semanal de dengue. Cuernavaca, Morelos: Servicios de Salud del estado de Morelos, 2006.

4. SSM. Registro epidemiológico semanal de dengue. Cuernavaca, Morelos: Servicios de Salud de Morelos, 2008.

5. Clark W, Parson E. Assessing vulnerability to global environmental risks. Cambridge MA: Belfer Center for Science and International Affairs, John F. Kennedy School of Government, Harvard University, 2000. 6. De Sherbinin A, Schiller A, Pulsipher A. The vulnerability of global cities to climate hazards. Environ Urban 2007;19(I):39-64.

7. Setbon M, Raude J. Population response to the risk of vector-borne diseases: lessons learned from socio-behavioural research during largescale outbreaks. Emer Health Threats J 2009;2.

8. INEGI. Censo poblacional. México: Instituto Nacional de Estadística y Geografía, 2005.

9. Hernandez B, Velasco H. Encuestas transversales. Epidemiología. Diseño y análisis de estudios. Mexico: INSP Editorial Medica Panamericana, 2009.

I0. Johnson DE. Métodos multivariados aplicados al análisis de datos. México: International Thomson Editores, 2000.

II. Jolliffe I. Principal component analysis. New York: Springer-verlag, 1996.

12. Camponovo MG. Analysis of the Uruguayan population vulnerability to dengue due to climate changes. Epidemiology 2008; 19 (I):S230 210.1097/1001.ede.0000291938.0000245387.0000291921.

13. Cutter S, Boruff B, Shirley W. Social vulnerability to environmental hazards. Social Science Quarterly 2003;84(2):242-26I.

14. Moro M, Gagliotti C, Silvi G, Angeli R, Sambri V, Rezza G et al. Knowledge, attitudes and practices survey after an outbreak of chikungunya infections. Int Health 20I0;2(3):223-227.

15. Heintze C, Garrido MV, Kroeger A. What do community-based dengue control programmes achieve? A systematic review of published evaluations. Trans R Soc Trop Med Hyg 2007; I0I (4):3 17-325.
16. Cifuentes E. Factores ambientales que determinan la aparición de brotes y la persistencia del dengue en Morelos. Salud Publica Mex 2007;29:4.

17. Toledo-Romaní ME, Boelaert M, Baly-Gil A, Ceballos-Ursula E, Van der Stuyft P. Participación comunitaria en la prevención del dengue: un abordaje desde la perspectiva de los diferentes actores sociales. Salud Publica Mex. 2006;48(I).

18. Van Benthem B, Khantikul N, Panart K, Kessels P, Somboon P, Oskam L. Knowledge and use of prevention measures related to dengue in northern Thailand. Trop Med Int Health 2002;7(II):993-1000.

19. Winch P, Lloyd L, Godas M, Kendall C. Beliefs about the prevention of dengue and other febrile illnesses in Mérida, Mexico. Am J Trop Med Hyg 1991;94(6):377.

20. Romero A, Martínez C, Cano S, Jiménez A. Impacto de las acciones de promoción y control en la prevención del dengue. Salud en Tabasco 2005; II (3):38I.

21. Soto HR, Fernández C, Ávila M. Evaluación de un programa educativo sobre dengue y Aedes aegypti focalizado en niños de escuela primaria. Rev Med Hondur 1995;63(I): 12 .

22. Navarrete J, Acevedo J, Huerta E, Torres J, Gavaldón D. Prevalencia de anticuerpos contra dengue y leptospira en la población de Jáltipan, Veracruz. Salud Publica Mex 2006;48(3):220-228.

23. Suárez R, González C, Carrasquilla G, Quintero J. An ecosystem perspective in the socio-cultural evaluation of dengue in two Colombian towns. Cad Saude Publica. 2009;25:I04-I I4.

24. Montero G, Morera H, Ramírez A, Sanabria L. Percepción de vulnerabilidad y severidad de la enfermedad del dengue en la comunidad de Metrópolis II de Pavas, San José, Costa Rica. Rev Costarric Salud Publica 2008; 17:32.

25. Ko Y, Chen M, Yeh S. The predisposing and protective factors against dengue virus transmission by mosquito vector. Am J Epidemiol |992;|36(2):2|4. 